Europhysics Letters

PREPRINT

\title{
Domain Growth in Random Magnets
}

\author{
Raja Paul ${ }^{1}$, Sanjay Puri ${ }^{2}$ and Heiko Rieger ${ }^{1}$ \\ 1 Theoretische Physik, Universität des Saarlandes-66041 Saarbrücken, GERMANY. \\ 2 School of Physical Sciences, Jawaharlal Nehru University, New Delhi-110067, INDIA.
}

PACS. 75.40 - Dynamical properties, dynamical scaling, numerical simulation.

PACS. 05.50.+q - Lattice theory and statistics (Ising, Potts, etc.).

PACS. 75.10.Nr - Spin-glass and other random models.

\begin{abstract}
We study the kinetics of domain growth in ferromagnets with random exchange interactions. We present detailed Monte Carlo results for the nonconserved random-bond Ising model, which are consistent with power-law growth with a variable exponent. These results are interpreted in the context of disorder barriers with a logarithmic dependence on the domain size. Further, we clarify the implications of logarithmic barriers for both nonconserved and conserved domain growth.
\end{abstract}

A homogeneous binary mixture becomes thermodynamically unstable if it is rapidly quenched below the coexistence curve. The subsequent far-from-equilibrium evolution of the system is characterized by the emergence and growth of domains enriched in the new equilibrium phases. The domain morphology is quantified by (a) the time-dependence of the domain scale $R(t)$, where $t$ is the time after the quench; and (b) the correlation function or its Fourier transform, the structure factor [1]. There is a good understanding of domain-growth kinetics in pure and isotropic systems, where the domain scale shows a power-law behavior, $R(t) \sim t^{\theta}$. For the case with nonconserved order parameter, e.g., ordering of a ferromagnet into up and down phases, we have $\theta=1 / 2$. On the other hand, for the case with conserved order parameter, e.g., phase separation of a binary $(\mathrm{AB})$ mixture into A- and B-rich domains, we have $\theta=1 / 3$ when growth is driven by diffusion.

Of course, real experimental systems are neither pure nor isotropic. In this letter, we focus on domain growth in ferromagnets and binary alloys with quenched disorder in the form of random exchange interactions. There have been many experimental [2-4] and numerical [5-10] studies of this problem [11]. At early times, domain coarsening is not affected by disorder. Then, there is a crossover to a disorder-affected regime, which occurs earlier for higher disorder amplitudes. There have also been many studies of domain growth in spin glasses [12,13], where the amplitude of disorder is such that the local exchange coupling may be either ferromagnetic or antiferromagnetic. Inspite of this attention, the nature of asymptotic domain growth in both random magnets and spin glasses remains the subject of much controversy. The present letter resolves this controversy in the context of random magnets. We present detailed Monte Carlo (MC) results which show that asymptotic growth in these systems is consistent with a power-law behavior, with the growth exponent $(\theta)$ depending on the temperature $(T)$ and

(c) EDP Sciences 
disorder amplitude $(\epsilon)$. Further, we present analytical arguments which clarify the functional form of $\theta(T, \epsilon)$ for systems with both nonconserved and conserved order parameters.

We study the random-bond Ising model (RBIM) with the following Hamiltonian:

$$
H=-\sum_{\langle i j\rangle} J_{i j} S_{i} S_{j}, \quad S_{i}= \pm 1,
$$

where the exchange couplings $J_{i j}$ are drawn from a probability distribution, e.g., Gaussian, uniform, bimodal, etc. We focus on the nearest-neighbor case, denoted by the subscript $\langle i j\rangle$. A kinetic version of the RBIM is obtained by associating Glauber spin-flip kinetics $\left(S_{i} \rightarrow-S_{i}\right)$ or Kawasaki spin-exchange kinetics $\left(S_{i} \leftrightarrow S_{j}\right)$ with the Hamiltonian in Eq. (1). In this letter, we consider the ferromagnetic case, where $J_{i j}>0$ always. The case where $J_{i j}$ can be both $>0$ (ferromagnetic) and $<0$ (antiferromagnetic) is relevant to spin glasses.

An important study of the nonconserved RBIM is due to Huse and Henley (HH) [15]. $\mathrm{HH}$ argued that coarsening domains are trapped by energy barriers $E_{B}(R) \simeq E_{0} R^{\psi}$, with exponent $\psi=\chi /(2-\zeta)$, where $\chi$ and $\zeta$ are the pinning and roughening exponents. For $d=2$, these exponents are known to be $\chi=1 / 3$ and $\zeta=2 / 3$ [16], yielding $\psi=1 / 4$. For $d=3$, a perturbative calculation gives $\psi \simeq 0.55$ [15]. To obtain the corresponding growth law, it is convenient to use the framework of Lai et al. (LMV) [17]. For curvature-driven growth in nonconserved systems, the length scale obeys

$$
\dot{R}=a(R, T) / R,
$$

where $a(R, T)$ is the diffusion constant which depends (in general) upon both the length scale and the temperature. In the presence of energy barriers, domain growth proceeds via thermally-activated barrier hopping with $a(R, T) \simeq a_{0} \exp \left(-E_{B} / T\right)$. Replacing the $\mathrm{HH}$ energy-barrier scaling in Eq. (2), we obtain the crossover behavior:

$$
\begin{gathered}
R(t) \simeq R_{0}(T, \epsilon) h\left(\frac{t}{t_{0}}\right), \quad \text { with } \\
R_{0}(T, \epsilon)=\left(\frac{T}{E_{0}}\right)^{1 / \psi}, \quad t_{0}(T, \epsilon)=\frac{1}{a_{0} \psi}\left(\frac{T}{E_{0}}\right)^{2 / \psi}, \quad \text { and } \\
h(x)=\left(\frac{2}{\psi} x\right)^{1 / 2}, \quad x \ll 1, \\
=(\ln x)^{1 / \psi}, \quad x \gg 1 .
\end{gathered}
$$

The logarithmic growth law proposed by $\mathrm{HH}$ has motivated many experimental and numerical studies of domain growth in random magnets. However, to date, there is no clear confirmation of this growth law. For example, the experiments of Ikeda et al. [2] found that there is no universal logarithmic law. Numerical studies have also found no evidence for a HH-type law, valid over extended time-windows and parameter regimes. On the contrary, some experiments [4] and simulations [6] suggest power-law growth with a variable exponent.

Let us next present results from our MC simulations, which were done on $L \times L$ lattices (in $d=2$ ) with periodic boundary conditions. The results presented here were obtained for uniform $J_{i j}$-distributions on the interval $[1-\epsilon / 2,1+\epsilon / 2]$ or $\left[1-\epsilon^{\prime}, 1\right]$, where $\epsilon$ and $\epsilon^{\prime}$ quantify the degree of disorder. The limits $\epsilon=0$ (or $\left.\epsilon^{\prime}=0\right)$ and $\epsilon=2\left(\right.$ or $\left.\epsilon^{\prime}=1\right)$ correspond to 

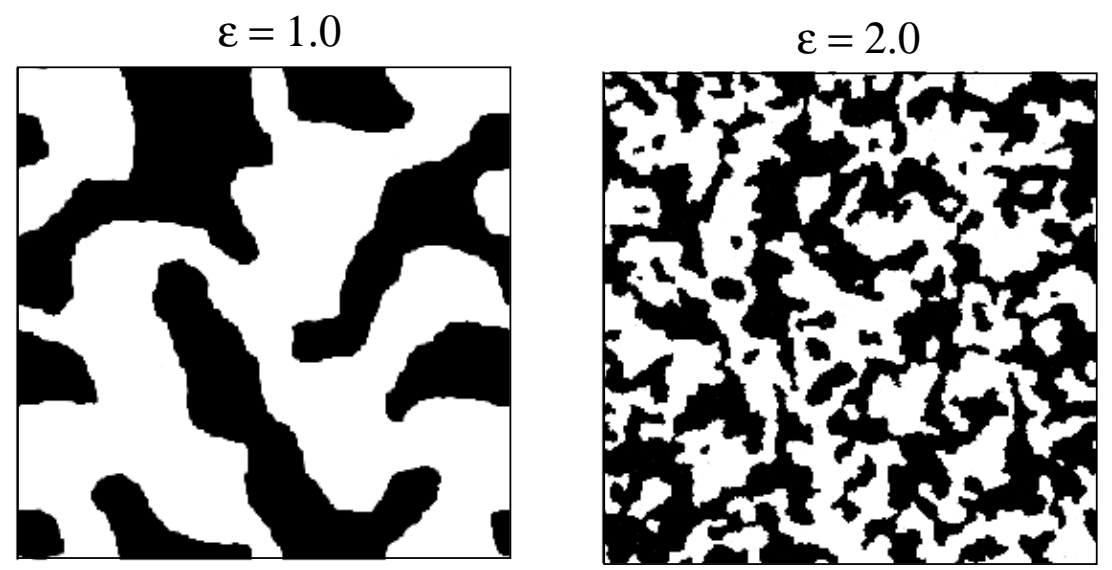

Fig. 1 - Domain growth in the RBIM with Glauber kinetics. We show evolution pictures at $t=10^{5}$ MCS for a $512 \times 512$ lattice, after a quench from $T=\infty$ to $T=0.5$. The up spins are marked in black, and the down spins are unmarked. The snapshots correspond to different disorder amplitudes, $\epsilon=1,2$.

zero and maximum disorder, respectively. Assigning random initial orientations to each spin, we rapidly quench to $T<T_{c} \simeq 2.269$. All our statistical data is obtained with lattice sizes $L=1024$, as an average over 50 independent initial conditions and disorder configurations. We also produced data for $L=256$ and 512 (not shown) to ensure that our results are not influenced by finite size effects.

Figure 11 shows typical evolution snapshots (at $t=10^{5} \mathrm{MCS}$ ) for different disorder amplitudes $\epsilon=1,2$. As expected, the length scale at a given time decreases with increase in $\epsilon$. However, the domain morphology does not visually differ from that for the pure case. We have confirmed (not shown here) that the scaled correlation function $[C(r, t)$ vs. $r / R(t)]$ is independent of the disorder amplitude, and is numerically equivalent to that for the pure case $(\epsilon=0)$. This observation has earlier been made by Puri et al. [8] and Bray and Humayun [9].

Next, we consider the time-dependence of the domain size, which is obtained as the distance over which the correlation function decays to half its maximum value. In Fig. 2] we undertake a direct test of the HH law by first plotting. $R^{1 / 4}$ vs. $t$ on a log-linear scale for different quench temperatures and $\epsilon^{\prime}=1$, with $J_{i j} \in\left[1-\epsilon^{\prime}, 1\right]$. The data does not satisfy the asymptotic $\mathrm{HH}$ growth law, which corresponds to a straight line on this plot. More generally, we have attempted to fit the data to the logarithmic function, $\ln t=a R^{x}+b$. This function does not give a good fit to the data. For acceptable fits, the exponent $x$ depends strongly on the temperature (cf. Ref. [2]), in contrast with the prediction of a universal growth law. Similar results are obtained if we fix $T$ and vary $\epsilon$, and we do not present these here.

We also tried to fit the data sets to the crossover scaling form described by Eqs. (3)-(5), the result for which is shown in the right portion of Fig. 2. We record the following points of disagreement with the proposed scaling: (1) The short-time behavior is not described well by Eq. (5), i.e., in the plot $g(x)$ does not fit well to the scaling curve. (2) The proposed asymptotic behavior, i.e., the curve $f(x)$, does not fit the scaling curve well even for the largest times. (3) The temperature-dependence of the crossover length $R_{0}(T)$ and the crossover time $t_{0}(T)$ is stronger than a power law, which is incompatible with the expected behavior in Eq. (4). The parameters $E_{0}$ and $a_{0}$ occurring in Eq. (4) are expected to decrease with increasing temperature, and therefore $R_{0}$ and $t_{0}$ may be expected to increase faster than $T^{4}$ and $T^{8}$, 

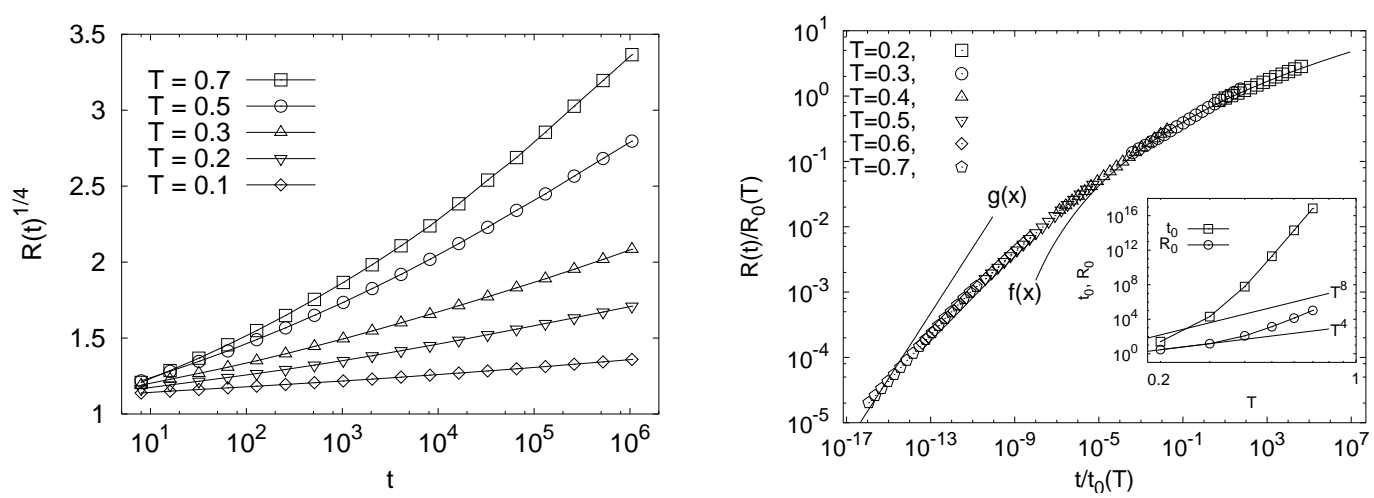

Fig. 2 - Left: Plot of $R^{1 / 4}$ vs. $t$ on a log-linear scale for $\epsilon^{\prime}=1$, i.e. $J_{i j}$ is uniformly distributed between $[0,1]$, and different quench temperatures. We show data for 5 temperature values: $T=$ 0.1, 0.2, 0.3, 0.5, 0.7. Right: Scaling plot according to Eqs. (3)-(5) ( $J_{i j}$ as in left figure). For each temperature $T$ the values for $R_{0}(T)$ and $t_{0}(T)$ have been chosen so as to obtain a smooth scaling curve $h(x)$. The functions $g(x) \propto x^{1 / 2}$ and $f(x) \propto(\ln x)^{4}$ represent the expected asymptotic behaviors for $x \ll 1$ and $x \gg 1$, respectively, according to Eq. (5). The inset shows the temperature-dependence of the fit values $R_{0}(T)$ and $t_{0}(T)$ and their expected $T$-dependence according to Eq. (4), which is $T^{4}$ and $T^{8}$, respectively, if $a_{0}$ and $E_{0}$ are only weakly (sublinearly) $T$-dependent.

respectively. However, their putative $T$-dependence turns out to be much too strong. Note that the crossover time $t_{0}$ in the inset of Fig. 2 varies over 20 decades when $T$ varies over only half a decade from 0.2 to 0.7 , and we do not see why the pinning energy amplitude $E_{0}$ or the domain wall tension should have such a strong $T$-dependence. Based on observations 1-3, we believe that the crossover scaling form in Eqs. (3)-(5) does not describe the data well and we suggest here an alternative picture.

Several groups $[4,6]$ have reported that random magnets exhibit power-law growth with variable exponents. In Fig. 3 (left), we plot $R$ vs. $t$ on a log-log scale for the data in Fig. 2 For low $T$, the plots are linear over 5 decades in time. For high $T$, the $\log$-log plots exhibit
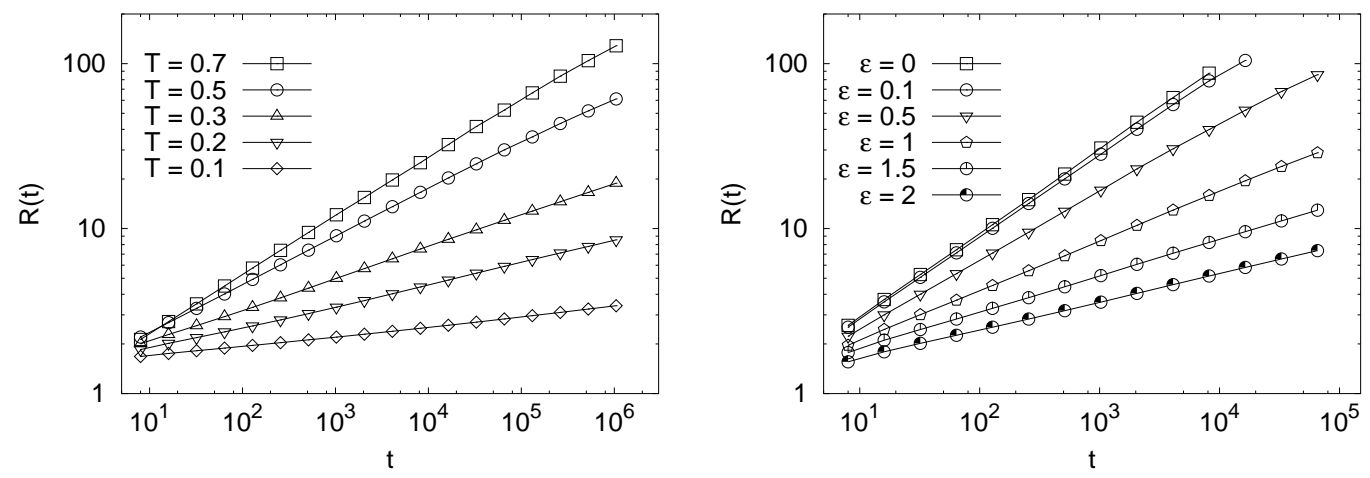

Fig. 3 - Left: Log-log plot of $R$ vs. $t$ for the length-scale data shown in Fig. 2 Right: Log-log plot of $R$ vs. $t$ for $T=0.5$ and different disorder amplitudes: $\epsilon=0$ (pure case), and $\epsilon=0.1,0.5,1,1.5,2$. Here $J_{i j}$ is uniformly distributed over $[1-\epsilon / 2,1+\epsilon / 2]$. 
a crossover behavior. In Fig. 3 (right), we present a similar plot for $T=0.5$, and different values of the disorder. Again, the data exhibits a power-law behavior for large values of $\epsilon$, and crossover behavior for small values of $\epsilon$. Our data is consistent with power-law growth with a variable exponent, at least for low $T$ and high $\epsilon$. We have obtained similar results for domain growth in the dilute Ising model (DIM), and will present these elsewhere [21].

Let us understand the origin of growth exponents which depend on $T$ and $\epsilon$. For the DIM, Henley [18] and Rammal and Benoit [19] have argued that the fractal nature of domain boundaries results in a logarithmic $R$-dependence of trapping barriers. We propose that this is generally applicable [14] and examine the implications thereof. At early times and small length scales, we expect disorder-free domain growth. This suggests the barrier-scaling form:

$$
E_{B}(R) \simeq \epsilon \ln (1+R)
$$

where $R$ is measured in dimensionless units. Substituting $a(R, T) \simeq a_{0} \exp \left(-E_{B} / T\right)$ in Eq. (2), we obtain

$$
\dot{R}=a_{0}(1+R)^{-\epsilon / T} / R
$$

The solution of Eq. (17) is the crossover function

$$
\begin{aligned}
R(t) & \simeq\left(2 a_{0} t\right)^{1 / 2}, \quad t \ll t_{0}, \\
& \simeq\left[(2+\epsilon / T) a_{0} t\right]^{\theta(T, \epsilon)}, \quad t \gg t_{0},
\end{aligned}
$$

with the growth exponent

$$
\theta(T, \epsilon)=(2+\epsilon / T)^{-1}
$$

The early- and late-time behaviors in Eq. (18) arise in the limits $R \ll 1$ and $R \gg 1$, respectively.

The crossover length and time can be identified by reformulating Eq. (8) as Eq. (3) with

$$
\begin{aligned}
& R_{0}(T, \epsilon)=\frac{1}{(2 \theta)^{\theta /(1-2 \theta)}}, t_{0}(T, \epsilon)=\frac{1}{a_{0}} \frac{1}{\left(2 \theta^{2 \theta}\right)^{1 /(1-2 \theta)}}, \quad \text { and } \\
& h(x)=x^{1 / 2}, \quad x \ll 1 \\
&=x^{\theta}, \quad x \gg 1 .
\end{aligned}
$$

In Fig. 4 we plot $1 / \theta$ vs. $1 / T$ and $\epsilon$ for the data shown in Fig. 3 The resultant linear plots confirm the logarithmic barrier-scaling scenario proposed above. For high temperatures and weak disorder amplitudes, recall that the length-scale data exhibits a crossover behavior. In these cases, we estimate the asymptotic exponent by tracking the effective exponent $\theta_{\text {eff }}=$ $d(\ln R) / d(\ln t)$ as a function of time.

We note the following points: (1) When measuring the growth by alternative methods, we get the same estimates for the growth exponents. For instance, we applied the following Monte Carlo renormalization group approach. First, we scaled the lattice twice by taking 4 block spins to reduce thermal noise. As a result, a $1024^{2}$ lattice becomes a $256^{2}$ lattice. Then we scanned horizontally and vertically through the lattice and measured the total number of domain walls. Finally, we divided the system area by this number to get the average domain length. From the time-dependence, we extracted the exponents $\theta$ shown in Fig. 4 (left), and we observe that they agree well within numerical accuracy. (2) We checked that 

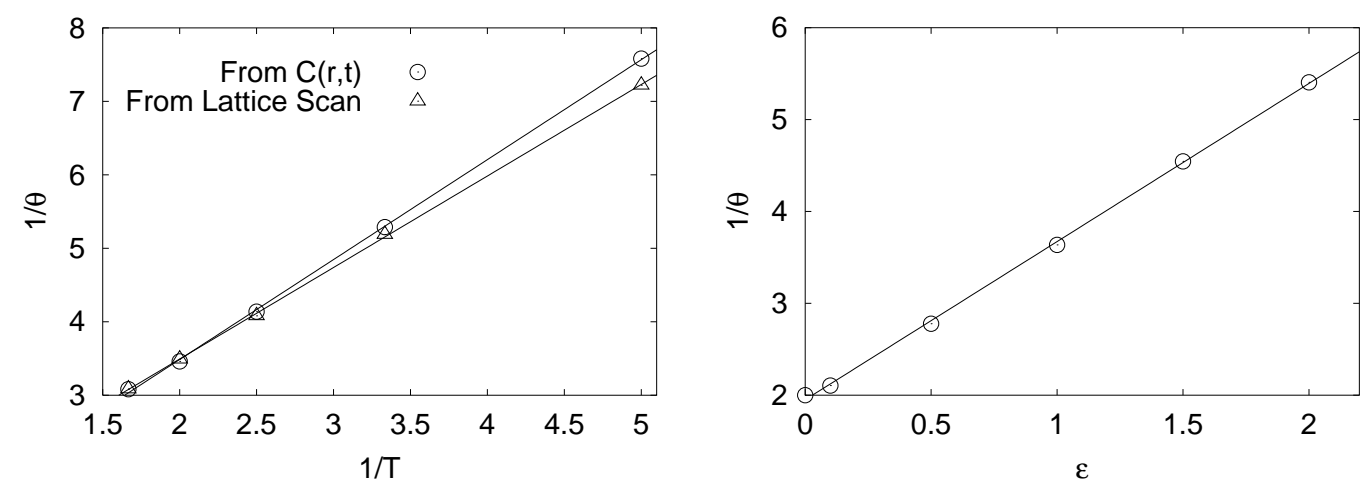

Fig. 4 - Left: Exponent $1 / \theta$ vs. $1 / T$ for the data shown in the left part of Fig. 3 (circles), and obtained for the same systems using estimates for the domain size via the lattice scaling method described in the text (squares). Note that for $T>1 \approx T_{c}\left(\epsilon^{\prime}=1\right)$, i.e., for $1 / T<1$ the system is in the paramagnetic phase. Hence $R$ cannot grow algebraically any more and no data for $1 / \theta$ are available in this region. Right: Exponent $1 / \theta$ vs. $\epsilon$ for the data shown in the right part of Fig. 3

the correlation functions that we calculated obey the expected dynamical scaling behavior: $C(r, t)=c[r / R(t)]=c\left[r / t^{\theta(T, \epsilon)}\right]$.

Given our results in the nonconserved case, we expect that the length-scale data for the conserved case should also exhibit power-law behavior with a variable exponent. We have undertaken MC simulations of the RBIM with conserved kinetics, and the results will be presented elsewhere [21]. Here, we confine ourselves to discussing the implications of logarithmic energy barriers for growth exponents in conserved systems.

In the absence of disorder, the domain scale obeys the Huse equation [20] $\dot{R}=D_{0} / R^{2}$, with the solution $R(t) \simeq\left(3 D_{0} t\right)^{1 / 3}$, which is referred to as the Lifshitz-Slyozov growth law. As before, the presence of disorder renormalizes the diffusion constant $D_{0}$ by an Arrhenius factor. For logarithmic barriers as in Eq. [6], the corresponding kinetic equation is

$$
\dot{R}=D_{0}(1+R)^{-\epsilon / T} / R^{2} .
$$

The short- and long-time solutions of Eq. (12) are obtained as follows: $R(t) \simeq\left(3 D_{0} t\right)^{1 / 3}$ for $t \ll t_{0}$ and $R(t) \simeq\left[(3+\epsilon / T) D_{0} t\right]^{\theta(T, \epsilon)}$ for $t \gg t_{0}$, where

$$
\theta(T, \epsilon)=(3+\epsilon / T)^{-1} .
$$

The asymptotic exponent differs from that for the nonconserved case. This should be contrasted with the HH scenario, where the asymptotic growth law is the same for the nonconserved and conserved cases $[1,8]$, which is seen by incorporating the HH barrierscaling form in $\dot{R}=D_{0} / R^{2}$. The crossover form of the solution of Eq. (12) is Eq. (3) with $R_{0}(T, \epsilon)=1 /(3 \theta)^{\theta /(1-3 \theta)}$ and $t_{0}(T, \epsilon)=D_{0}^{-1} /\left(3 \theta^{3 \theta}\right)^{1 /(1-3 \theta)}$ and $h(x)=x^{1 / 3}$ for $x \ll 1$ and $h(x)=x^{\theta}$ for $x \gg 1$.

In conclusion, we have presented detailed MC results for domain growth in random magnets for a wide range of temperatures and disorder amplitudes. Our results do not support the $\mathrm{HH}$ scenario of logarithmic domain growth. (Of course, we cannot rule out the possibility of a logarithmic regime at even later times than those investigated here.) Rather, they are in agreement with results $[4,6]$ which exhibit power-law growth with a variable exponent. This 
scenario arises naturally in the context of logarithmic energy barriers, and the corresponding functional dependence of $\theta$ on $T$ and $\epsilon$ is in excellent agreement with our numerical results. The results in this letter provide a framework for the analysis of experiments and simulations on domain growth in disordered magnets and binary mixtures.

This work was financially supported by the Deutsche Forschungsgemeinschaft (DFG), SFB277.

\section{REFERENCES}

[1] Bray A. J., Adv. Phys., 43 (1994) 357;

Binder K. and Fratzl P., Materials Science and Technology, edited by G. Kostorz, Vol. 5 (Wiley-VCH, Weinheim) 2001, p. 409;

Dattagupta S. and Puri S., Dissipative Phenomena in Condensed Matter Physics, Springer Series in Materials Science, Vol. 71 (Springer-Verlag, Heidelberg) 2003.

[2] Ikeda H., Endoh Y. and Iтон S., Phys. Rev. Lett., 64 (1990) 1266.

[3] Schins A. G., Arts A. F. M. and De Wijn H. W., Phys. Rev. Lett., 70 (1993) 2340; Shenoy D. K., Selinger J. V. and Grüneberg K. A. and Naciri. J. and Shashidhar R., Phys. Rev. Lett., 82 (1999) 1716.

[4] Likodimos V., Labardi M. and Allegrini M., Phys. Rev. B, 61 (2000) 14440; Likodimos V., Labardi M., Orlik X. K., Pardi L., Allegrini M., Emonin S. and Marti O., Phys. Rev. B, 63 (2001) 064104.

[5] Grest G. S. and Srolovitz D. J., Phys. Rev. B, 32 (1985) 3014.

[6] Он J. H. and Choi D.-I., Phys. Rev. B, 33 (1986) 3448.

[7] Chowdhury D., Grant M. and Gunton J. D., Phys. Rev. B, 35 (1987) 6792; Chowdhury D. and Kumar S., J. Stat. Phys., 49 (1987) 855;

Biswal B., Puri S. and Chowdhury D., Physica A, 229 (1996) 72.

[8] Puri S., Chowdhury D. and Parekh N., J. Phys. A, 24 (1991) L1087; Puri S. and Parekh N., J. Phys. A, 25 (1992) 4127.

[9] Bray A. J. and Humayun K., J. Phys. A , 24 (1991) L1185.

[10] Gyure M. F., Harrington S. T., Strilka R. and Stanley H. E., Phys. Rev. E, 52 (1995) 4632.

[11] For a review, see Chapter 4 of Dattagupta and Puri [1].

[12] Young A. P., Spin glasses and random fields (World Scientific, Singapore) 1997.

[13] Kawashima N. and Rieger H., cond-mat, 0312432 (.)

[14] A logarithmic $R$ dependence of barriers was also proposed for spin glasses, see Rieger H., J. Phys. A, 26 (1993) L615;

Kisker J, Santen L., Schreckenberg M. and Rieger H., Phys. Rev. B, 53 (1996) 6418. Here it also leads to an algebraic growth law with temperature dependent exponents.

[15] Huse D. A. And Henley C. L., Phys. Rev. Lett, 54 (1985) 2708.

[16] Forster D., Nelson D.R. and Stephen M. J., Phys. Rev. A, 16 (1977) 732;

Huse D. A., Henley C. L. and Fisher D. S.,, Phys. Rev. Lett., 55 (1985) 2924.

[17] Lai Z. W., Mazenko G. F. and Valls O. T., Phys. Rev. B, 37 (1988) 9481.

[18] Henley C. L., Phys. Rev. Lett., 54 (1985) 2030.

[19] Rammal R. and Benoit A., Phys. Rev. Lett, 55 (1985) 649.

[20] Huse D. A., Phys. Rev. B, 34 (1986) 7845.

[21] Paul R., Puri S. and Rieger H., to be published. 